\title{
Luxación expuesta de rodilla: ¿Qué hemos aprendido en los últimos 20 años?
}

\section{Open Knee Dislocations: What have we learn about it in the last 20 years?}

\author{
G. Xabier Carredano1,2 $\quad$ R. Juanjose Valderrama ${ }^{1,3}$ G. Sergio Arellano ${ }^{2,4}$ B. Diego Montenegro ${ }^{5}$

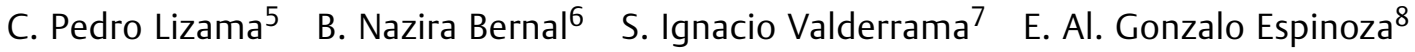 \\ L. Gonzalo Espinoza ${ }^{1,2}$
}

${ }^{1}$ Traumatología, Equipo de Cirugía de Rodilla y Artroscopía Mutual de

Address for correspondence Xabier Carredano Gonzalez, MD, Hospital Clínico Mutual de Seguridad C.Ch.C., Avenida Alameda Libertador Bernardo ÒHiggins 4848, Edificio A $4^{\circ}$ Piso, Estación Central, Santiago, Chile

2 Traumatología, Clínica Alemana de Santiago, Santiago, Chile

3 Traumatología, Equipo de Rodilla Clínica Indisa, Santiago, Chile

${ }^{4}$ Traumatología, Cirugía de Rodilla y Artroscopía Hospital Padre (e-mail: xcarredano@gmail.com; xcarredano@me.com).

${ }^{5}$ Residencia de Ortopedia y Traumatología Mutual de SeguridadUniversidad de Valparaíso, Valparaíso, Chile

${ }^{6}$ Residencia de Ortopedia y Traumatología UDD-Clínica Alemana de Santiago-Mutual de Seguridad, Santiago, Chile

${ }^{7}$ Medico Cirujano, Santiago, Chile

8 Interno de Medicina Universidad Católica de Chile, Santiago, Chile

Rev Chil Ortop Traumatol 2018;59:47-54.

\section{Resumen}

received

December 28, 2017

accepted

June 19, 2018

published online

August 10, 2018
Introducción La luxación expuesta de rodilla es compleja, de incidencia baja con grandes secuelas funcionales. Existen escasos reportes de series en la literatura relativos a su manejo y resultados.

Objetivo El objetivo de este trabajo fue describir los resultados obtenidos de todos los pacientes con luxación expuesta de rodilla tratados durante las últimas dos décadas en nuestro hospital bajo un mismo estándar de tratamiento.

Método Estudio descriptivo retrospectivo en una serie quirúrgica de 11 pacientes con luxación expuesta de rodilla, tratados entre 1994 y 2015. Todos fueron estudiados y manejados según esquema estandarizado: Angiografía/ angioTC, aseo quirúrgico, fijador externo y reparación neurovascular en casos necesarios. Revisión de registros clínicos e imagenológicos consignando datos demográficos, lesiones concomitantes, número y tipo de cirugías, y complicaciones asociadas. Seguimiento promedio fue de 10,7 años con evaluación mediante encuesta funcionales SF-12/IKDC durante el mes de marzo de 2015.

Resultados Diez pacientes eran hombres, 1 mujeres. Edad promedio al accidente 38,6 años. Mecanismo lesional de alta energía; como referencia la clasificación de Schenck, 1 lesión III-M, 4 tipo IV y los 6 restantes una tipo V. 4 lesiones vasculares (36.4\%) y 7 lesiones neurológicas (63.6\%). Tratamiento definitivo consistió en 4 reconstrucciones ligamentarias, 2 prótesis, 3 artrodesis y 2 amputaciones supracondíleas. Evaluaciones funcionales dieron 


\author{
Palabras Clave \\ - luxación expuesta de \\ rodilla \\ - alta energía \\ - complicaciones \\ - schenck
}

\section{Abstract}

\section{Keywords}

- open dislocation

- high energy trauma

- vascular lesion como resultados un puntaje promedio de 37 y 48,5 para SF-12 físico y mental respectivamente, y de 44,1 para IKDC.

Conclusión La luxación expuesta de rodilla es una lesión infrecuente, muy compleja, asociada a accidentes de alta energía, con complicaciones severas, lo que determina resultados funcionales relativamente malos. La estandarización permite sistematizar las distintas etapas de atención, racionalizar los recursos disponibles evitando la improvisación en momentos críticos, lo que podría incidir en la obtención de resultados.

Nivel de Evidencia IV Serie de casos.

Introduction Open knee dislocation is a complex lesion of low incidence and large functional sequelae. There are few series reports in the literature regarding its management and outcomes.

Objective Describe the results obtained from all patients with open knee dislocation treated during the last two decades in our hospital under the same treatment standard. Method Retrospective descriptive study in a surgical series of 11 patients with open knee dislocation, treated between 1994 and 2015. All were studied and managed according to a standardized protocol: Angiography/angioCT, surgical debridement, external fixation and neurovascular repair in necessary cases. Review of clinical and imaging records, recording demographic data, concomitant injuries, number and type of surgeries and associated complications. Average follow-up was 10.7 years, with an evaluation through functional surveys SF-12/IKDC during March 2015.

Results 10 patients were men, 1 woman. Average age at accident 38.6 years. High-energy trauma mechanism of injury; Schenk classification as reference, 1 lesion type III-M, 4 type IV and the remaining 6 type V. 4 vascular lesions (41.7\%) and 7 neurological lesions (63.6\%). Definitive treatment consisted in 4 ligamentous reconstructions, 2 prostheses, 3 arthrodesis and 3 supracondylar amputations. Functional evaluations showed an average score of 37 and 48.5 for SF-12 physical and mental respectively, and 44.1 for IKDC. Conclusion Open knee dislocation is a rare, very complex, associated to high-energy trauma, with severe complications, which determines relatively poor functional outcomes. Standardization allows to systematize the different stages of attention and rationalize available resources avoiding improvisation at critical moments, which could affect the results.

Level of evidence: IV Case series

\section{Introducción}

La incidencia reportada de luxación de la rodilla fluctúa entre 0,001\% y 0,013\% del total de lesiones ortopédicas. ${ }^{1-3}$ Debido al alto número de reducciones espontáneas en el sitio del accidente o durante el traslado a un centro asistencial, es probable que esos datos correspondan a una subestimación siendo su verdadera frecuencia desconocida ${ }^{2,4-6}$ Aun cuando en base a la información disponible se trata de una entidad infrecuente, es considerada una de las lesiones más graves de la rodilla debido al potencial compromiso de estructuras neurovasculares, complejos estabilizadores y fracturas asociadas al mecanismo traumático, pudiendo ser éste de alta o baja energía. ${ }^{1,3-5,7,8}$ La demora en el diagnóstico y tratamiento adecuado pueden determinar consecuencias devastadoras especialmente en aquellos casos asociados a lesión vascular que pone en riesgo la vitalidad de la extremidad. ${ }^{3,9}$
La luxación expuesta de rodilla corresponde a un sub grupo altamente infrecuente y de extrema complejidad cuya incidencia reportada fluctúa entre el 5\% y 17\% del total de luxaciones de rodilla, pudiendo corresponder a lesiones aisladas o con mayor frecuencia, en contexto de politraumatizados resultado de traumatismos de alta energía, escasamente reportados en la literatura. ${ }^{10,11}$

En este contexto, es imprescindible mantener un alto índice de sospecha en presencia de una lesión expuesta alrededor de la rodilla aun cuando ésta se encuentre reducida, dado el riesgo de presentar una lesión vascular que ponga en riesgo la extremidad y su función. ${ }^{11-15}$

Particularmente Natsuhara y col., reportan que el compromiso vascular en el contexto de esas lesiones determina un factor de mal pronóstico, llegando a un $20 \%$ de amputaciones en forma inicial y un $86 \%$ luego de revascularizaciones sobre 8 horas de evolución de isquemia. ${ }^{1}$ 
Debido a la baja incidencia reportada, la complejidad de esas lesiones y la escasez de estudios que permitan determinar la mejor conducta terapéutica, los resultados y alternativas de manejo son extremadamente variables. El objetivo de este trabajo fue describir los resultados obtenidos de todos los pacientes con luxación expuesta de rodilla tratados durante las últimas dos décadas en nuestro hospital bajo un mismo estándar de tratamiento.

\section{Materiales y Métodos}

Se realizó un estudio descriptivo, retrospectivo en una serie quirúrgica de pacientes con diagnóstico de luxación expuesta de rodilla entre los años 1994 y 2015, período en que se aplicó un esquema estandarizado para el manejo de esas lesiones. Durante ese tiempo, solamente se registraron 11 casos, a los cuales se les revisaron los registros clínicos, los estudios imagenológicos y adicionalmente se les aplicó escalas funcionales telefónicas.

Todos los pacientes ingresaron a nuestro centro a través del servicio de urgencia, donde se aplicó un esquema estandarizado por un equipo multidisciplinario compuesto por cirujano, traumatólogo, anestesista y personal paramédico, llevando a cabo la evaluación inicial según protocolo A.T.L.S.@ Durante la evaluación secundaria se realizó la exploración dirigida de la extremidad afectada en búsqueda de solución de continuidad de la piel, incongruencia articular, inestabilidad multidireccional y signos de lesión vascular (-Fig. 1). ${ }^{16}$

Posteriormente se continuó de acuerdo al siguiente esquema:

1. Profilaxis antibiótica: Cefalosporina $1^{\text {a }}$ gen aminoglucósido - penicilina

2. Evaluación vascular clínica: pulsos pedio y tibial posterior comparativo.

3. Imagenología inicial: Rx rodilla AP-L; angiografía (19941996) y angioTC EEII (1996-2015)
4. Reducción ortopédica: sala de reanimación o pabellón de urgencia.

5. Aseo quirúrgico - exploración - estabilización con fijador externo transarticular.

6. Lesión arterial: revascularización en aseo quirúrgico y estabilización inicial. (Cirujano vascular)

7. Aseos quirúrgico adicionales según evolución.

8. En todos los casos, se utilizó la clasificación descrita por Schenck para la categorización de las lesiones ligamentarias (- Tabla 1)..$^{17}$

\section{Técnica Quirúrgica}

El objetivo del tratamiento quirúrgico inicial de esas lesiones es preservar la extremidad, reparar las estructuras dañadas que lo permitan y realizar el cierre de las heridas traumáticas.

La reducción-osteosíntesis de fracturas y la reinserción de lesiones avulsivas junto con el tratamiento de los déficit de cobertura de las partes blandas, se realizó durante las primeras 2 semanas tras el accidente.

La reconstrucción de los ligamentos cruzados anterior(LCA) y posterior (LCP), junto con el tratamiento de lesiones intra articulares asociadas, fueron realizadas utilizando artroscopía la que se llevó a cabo bajo anestesia regional en decúbito supino con rodilla en flexión de $90^{\circ}$, soporte para muslo distal con tope medial-lateral y utilizando en todos los casos manguito de isquemia por un máximo de 120 minutos. Por su parte, las esquinas posteromedial (EPM) y posterolateral (EPL) fueron tratadas (reinserción-reconstrucción) en forma abierta. Durante los primeros años (1994-1998), se utilizaron autoinjertos y posteriormente aloinjertos para llevar a cabo las reconstrucciones.

\section{Tiempos Quirúrgicos-Rehabilitación}

Una vez estabilizados los pacientes fueron sometidos a cirugía de reconstrucción ligamentaria de acuerdo a la clasificación de la lesión; a las 2 semanas reconstrucción

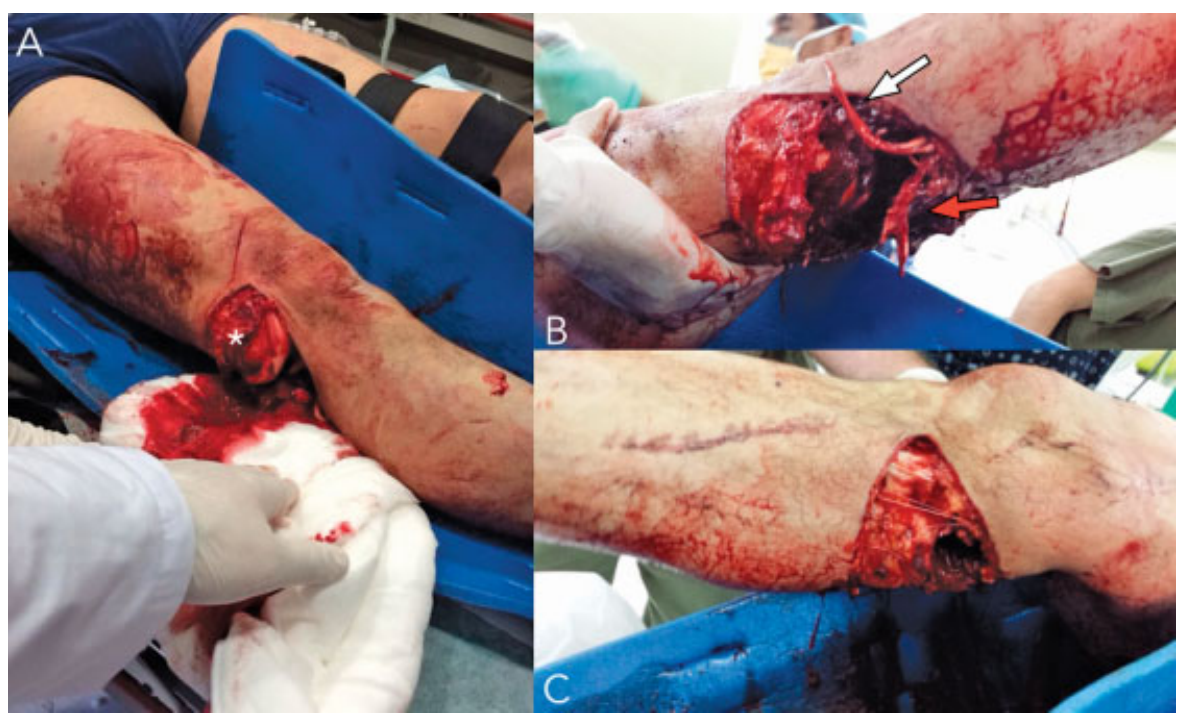

Fig. 1 Hombre, 42 años. Sufre atrapamiento de ambas extremidades inferiores entre ascensor y estructura metálica. Ingresa a Servicio de Urgencia con luxación expuesta de la rodilla derecha. La exposición del cóndilo femoral lateral $\left({ }^{*}\right)$ evidencia la persistencia de la pérdida de congruencia articular (A). Al realizar la exploración quirúrgica se evidencia la extensión de la exposición desde lateral (A) hasta medial (C) comprometiendo toda la región posterior (B), asociada a lesión completa de la arteria poplítea (flecha blanca) y nervio tibial (flecha roja). 
50 Luxación expuesta de rodilla Xabier Carredano y col

Tabla 1 Clasificación descrita por Robert Schenck

\begin{tabular}{|l|l|l|}
\hline \multicolumn{2}{|l|}{ Clasificación Anatómica de las Luxaciones de Rodilla (Schenck-2002) } \\
\hline Luxación GI & 1 cruzado +1 colateral & LCA + ligamento colateral \\
\cline { 2 - 3 } & & LCP + ligamento colateral \\
\hline Luxación GII & LCA + LCP & Colaterales intactos \\
\hline Luxación G III-M & LCA, LCP y LCM & EPL + LCL intactos \\
\hline Luxación G III-L & LCA, LCP y LCL + EPL & LCM intacto \\
\hline Luxación G IV & LCA, LCP, LCM y LCL + EPL & \\
\hline Luxación G V & Luxación + fractura & \\
\hline
\end{tabular}

Abreviaciones: EPL, esquinas posterolateral; EPM, esquinas posteromedial; LCA, ligamentos cruzados anterior; LCL, ligamentos cruzados lateral; LCM, ligamentos cruzados medial; LCP, ligamentos cruzados posterior.

del LCP, EPM, EPL y reparación del ligamento colateral medial (LCM); La reconstrucción del LCA fue diferida para un segundo tiempo después de las 12 semanas.
En todos los casos se inmovilizó la rodilla en extensión por un lapso de 4 semanas post cirugía; desde ese momento, se autorizó rango articular pasivo gradual hasta lograr $90^{\circ}$ de flexión, restringiendo el fortalecimiento de flexores y

Tabla 2 Resumen de datos de la serie

\begin{tabular}{|l|l|l|l|l|l|l|l|}
\hline $\mathbf{N}^{\mathbf{0}}$ & Sexo & Edad ACC & LAT & Neurológico & Vascular & CIR & Aseo \\
\hline 1 & H & 37 & IZQ & + C.P.E. & - & 5 & 2 \\
\hline 2 & H & 20 & DER & - & - & 3 & 1 \\
\hline 3 & H & 30 & IZQ & - & - & 9 & 7 \\
\hline 4 & H & 26 & DER & + C.P.E. & + A. Poplítea & 6 & 4 \\
\hline 5 & H & 49 & IZQ & - & - & 8 & 3 \\
\hline 6 & H & 26 & IZQ & + C.P.E. & + A. Poplítea & 11 & 4 \\
\hline 7 & H & 56 & IZQ & + C.P.E. & - & 6 & 3 \\
\hline 8 & H & 37 & DER & + C.P.E. & - & 6 & 1 \\
\hline 9 & H & 40 & DER & + C.P.E. & + A. Poplítea & 3 & 1 \\
\hline 10 & H & 44 & IZQ & + C.P.E. & - & 4 & 2 \\
\hline 11 & H & 52 & DER & - & + A. Poplítea & 6,1 & 3 \\
\hline & & & & & & 6,1 & 3 \\
\hline $\mathbf{N}^{\mathbf{0}}$ & Infec. & Cobertura & F.E. (días) & Seguimiento & SF-12 (P) & SF-12 (M) & IKDC \\
\hline 1 & - & - & 23 & 51 & 33,0 & 63,7 & 56,3 \\
\hline 2 & + & - & 62 & 42 & 30,6 & 41,9 & 36,8 \\
\hline 3 & + & + & 56 & 198 & 50,0 & 45,5 & - \\
\hline 4 & + & + & 39 & 68 & N/A & N/A & N/A \\
\hline 5 & + & - & 41 & 206 & 48,3 & 62,1 & 44,8 \\
\hline 6 & + & + & 67 & 243 & 30,6 & 41,9 & 37 \\
\hline 7 & + & + & 183 & 52 & 47,4 & 64,1 & 51,7 \\
\hline 8 & - & - & 56 & 136 & 44,2 & 56,4 & 59,8 \\
\hline 9 & - & - & 6 & 11 & 29,1 & 27,5 & - \\
\hline 10 & - & + & 74 & 222 & 26,0 & 55,4 & 39,1 \\
\hline 11 & - & + & 55 & 262 & 35,5 & 33,4 & 20,7 \\
\hline & 7 & & 172 & 37,5 & 49,2 & 43,3 \\
\hline
\end{tabular}

Abreviaciones: ACC, accidente; NC, número de cirugías; DER, derecho; FE, fijador externo; H, hombre; IKDC, International Knee Documentation Commitee; IZQ, izquierda; Infec., infección; LAT, lateralidad; M, mujer. 
manteniendo descarga absoluta hasta la $6^{\underline{a}}$ semana. Entre las $6^{\underline{a}}$ y $12^{\underline{a}}$ semanas el proceso de rehabilitación se centró en fortalecimiento muscular, propiocepción y aumento del rango articular. Tras completar 12 semanas y según edad, evolución y requerimientos, se realizó la reconstrucción del LCA.

Para la evaluación de resultados funcionales, se utilizaron las encuestas SF-12 (físico y mental) e IKDC (subjetivo), ambas realizadas telefónicamente durante el mes de junio de 2015, solicitando a los pacientes que respondieran evaluando su estado actual.

\section{Resultados}

Entre los años 1994 y 2015 un total de 11 pacientes (10 hombres y 1 mujer), consecutivos con luxación expuesta de rodilla secundaria en el $100 \%$ de los casos a traumatismos de alta energía (-Tabla 2), ingresaron y fueron tratados en nuestro hospital mediante la aplicación de un protocolo estandarizado esquematizado en -Fig. 2.

La edad promedio al momento del accidente fue de 38,6 años (rango de 20 a 59 años). La clasificación se realizó utilizando el sistema de categorización descrito por Schenck; según este esquema 6 casos (54,5\%) correspondieron a una luxación grado V, 4 (36,4\%) a grado IV y $1(9,1 \%)$ a grado III-M. La distribución y el número de estructuras ligamentarias comprometidas se detalla en la - Tabla 3.

Se realizaron en promedio 6 (rango 3 a 11) cirugías por cada paciente durante su tratamiento; entre ellas 3 en promedio (rango 1 a 7) correspondieron a aseos quirúrgicos.

La estabilización transitoria de la rodilla se realizó durante el aseo quirúrgico inicial con un fijador externo

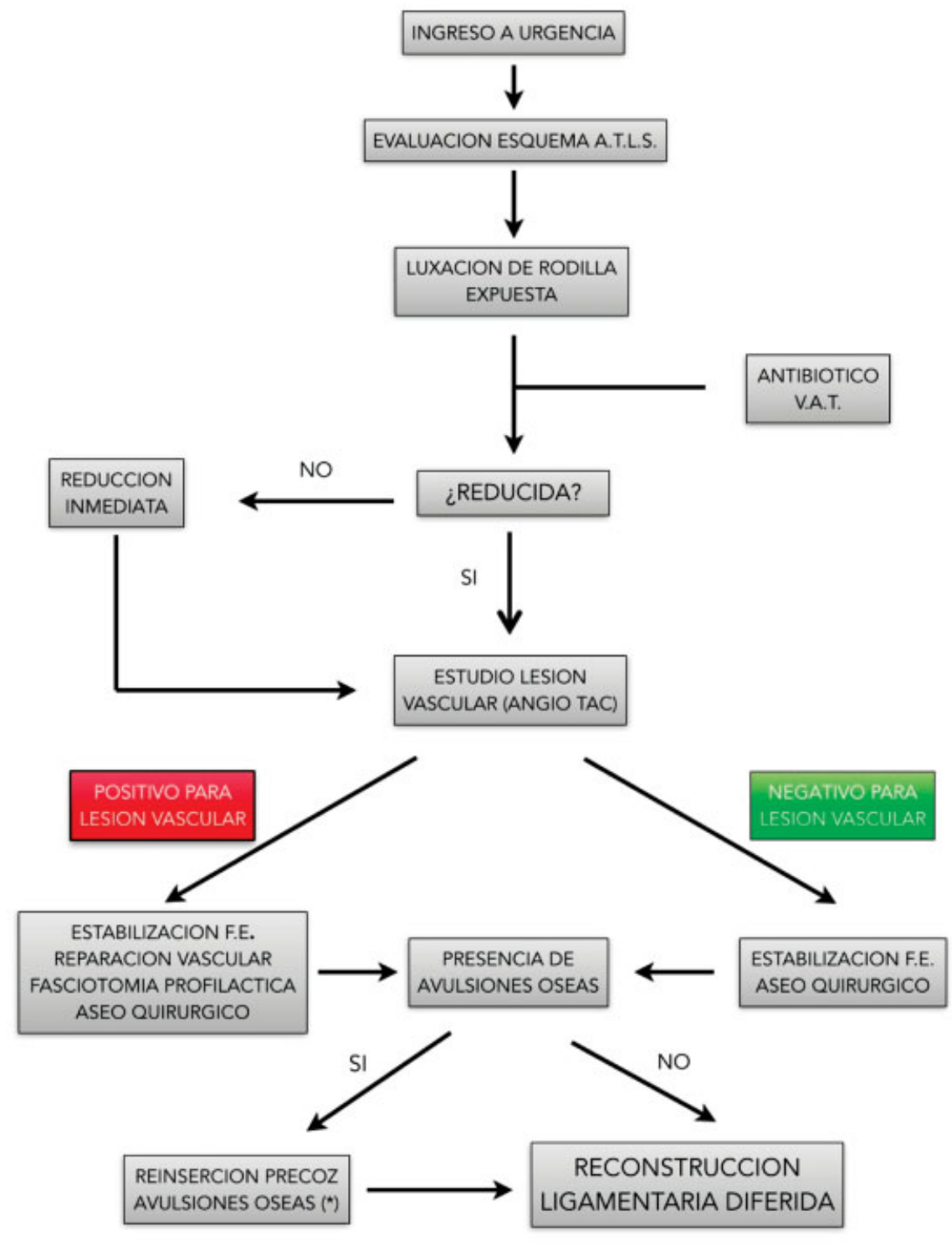

$\left({ }^{*}\right)<2$ SEMANAS

Fig. 2 Algoritmo de enfrentamiento estandarizado de pacientes con sospecha de luxación expuesta de rodilla. 
Tabla 3 Distribución de Icasos según complejos ligamentarios comprometidos

\begin{tabular}{|l|l|l|l|l|l|l|l|}
\hline Clasificación & $\mathrm{N}^{\circ}$ Casos & LCA & LCP & LCL & LCM & EPL & Fractura \\
\hline Grado I & 0 & & & & & & \\
\hline Grado II & 0 & & & & & & \\
\hline Grado III-M & 1 & + & + & & + & & \\
\hline Grado III-L & 0 & & & & & & \\
\hline Grado IV & 4 & + & + & + & + & + & \\
\hline Grado V & 6 & + & + & + & + & + & + \\
\hline Total & 11 & 11 & 11 & 10 & 11 & 10 & 6 \\
\hline
\end{tabular}

Abreviaciones: EPL, esquinas posterolateral; LCA, ligamentos cruzados anterior; LCL, ligamentos cruzados lateral; LCM, ligamentos cruzados medial; LCP, ligamentos cruzados posterior.

transarticular; el tiempo de uso promedio de este dispositivo fue de 60,2 días (rango 6 a 183 días).

El 36,4\% (4 casos) de los casos presentó lesión de la arteria poplítea con interrupción total del flujo sanguíneo a ese nivel. La reperfusión de la extremidad fue realizada por cirujano vascular mediante by pass fémoro tibial durante la cirugía inicial con buen resultado en la totalidad de los casos.

En relación a las lesiones neurológicas el 63,6\% (7 casos), presentó compromiso del nervio peroneo común, en 6 casos correspondió a neuropraxia y en uno a axonotmesis.

El déficit de cobertura de partes blandas afectó al 54,5\% (6 casos) de los casos; la evaluación y tratamiento de esas lesiones fue realizada por un cirujano plástico entrenado mediante el uso de colgajos e injertos tras objetivar ausencia clínica de infección local.

La infección fue la complicación más frecuente en esa serie al analizar su evolución a mediano-largo plazo afectando al $63,6 \%$ del total. Todos los casos fueron tratados utilizando antibióticos; 3 requirieron un aseo quirúrgico. Dentro de ese grupo, el 42,9\% (3 casos), correspondió a una osteomielitis crónica de la tibia.

En cuanto al tratamiento definitivo 18,2\% (2 casos) de los casos debió ser sometido a amputación de la extremidad afectada a nivel supracondíleo del fémur; uno de ellos a consecuencia de alteraciones funcionales derivadas de lesión neurológica y el segundo por presencia de osteomielitis crónica de la tibia. El 27,2\% (3 casos) fue tratado mediante artrodesis de la rodilla por rigidez y extenso compromiso de partes blandas. Al momento de la revisión de datos el 18,2\% (2 casos) de los casos fue sometido a cirugía de reemplazo articular con prótesis total de rodilla. (ATR).

La aplicación de las encuestas funcionales mostró un promedio de 37,0 puntos (26-50) y 48,5 puntos $(27,5-64,1)$ en el SF-12 físico y SF-12 Mental respectivamente. Por su parte el International Knee Documentation Comitee Score Subjetivo (I.K.D.C.s) dio como promedio 44,1 (20.7-59.8). Los resultados se resumen en la - Tabla 4.

Finalizado el tratamiento un $72,7 \%$ de los pacientes, reinició actividad laboral remunerada, siendo en todos los casos en cargos de menor exigencia física.

\section{Discusión}

La luxación traumática de rodilla es tan infrecuente como grave $\mathrm{y}$ se asocia a importantes secuelas físicas y funcionales. ${ }^{18}$ Históricamente, su tratamiento consistía en inmovilización prolongada con malos resultados clínicofuncionales determinados en su mayoría por pérdida de rango de movilidad presencia de inestabilidad residual y funcionalidad limitada. ${ }^{19}$

Lesiones de la arteria y vena poplítea pueden tener consecuencias devastadoras para los pacientes, por ello exigen diagnóstico y tratamiento precoz. ${ }^{10}$ La incidencia reportada de lesión vascular que requiere reparación fluctúa entre el $8 \%$ y el 22\% de las luxaciones traumáticas cerradas de rodilla y llega al 26\%-29\% en el caso de luxaciones expuestas. $^{1,10,16,20}$ Nuestra serie presenta un porcentaje mayor al descrito lo que podría estar relacionado con el elevado nivel de energía involucrada en todos los casos y el reducido número total de casos recibidos durante las últimas 2 décadas.

Se ha descrito el uso del Índice tobillo-brazo (ABI) en conjunto con el examen físico para determinar la necesidad de realizar angiografía-angioCT. ${ }^{18}$ Mills y col., reportaron sensibilidad, especificidad y valor predictivo positivo (VPP) de $100 \%$ con $\mathrm{ABI}<0,9$ para la detección de lesión vascular. ${ }^{18} \mathrm{Su}$ aplicación requiere de personal entrenado permanentemente en servicio de urgencia, recurso no disponible en nuestro medio $\mathrm{y}$ un resultado no concluyente obliga a realizar estudio imagenológico vascular. Por esa razón, ABI no está incluido en el algoritmo de nuestro centro siendo reemplazado por angioCT en la evaluación de urgencia (-Fig. 3).

Tabla 4 Resumen Resultados Evaluación Funcional

\begin{tabular}{|l|l|l|}
\hline & Promedio & Rango \\
\hline SF 12 PCS & 37 & $26-50$ \\
\hline SF 12 MCS & 48,5 & $27.5-64.1$ \\
\hline IKDC & 44,1 & $20.7-59.8$ \\
\hline
\end{tabular}

Abreviaciones: IKDC, International Knee Documentation Committee; MCS, mental component summary; PCS, physical component summary. 


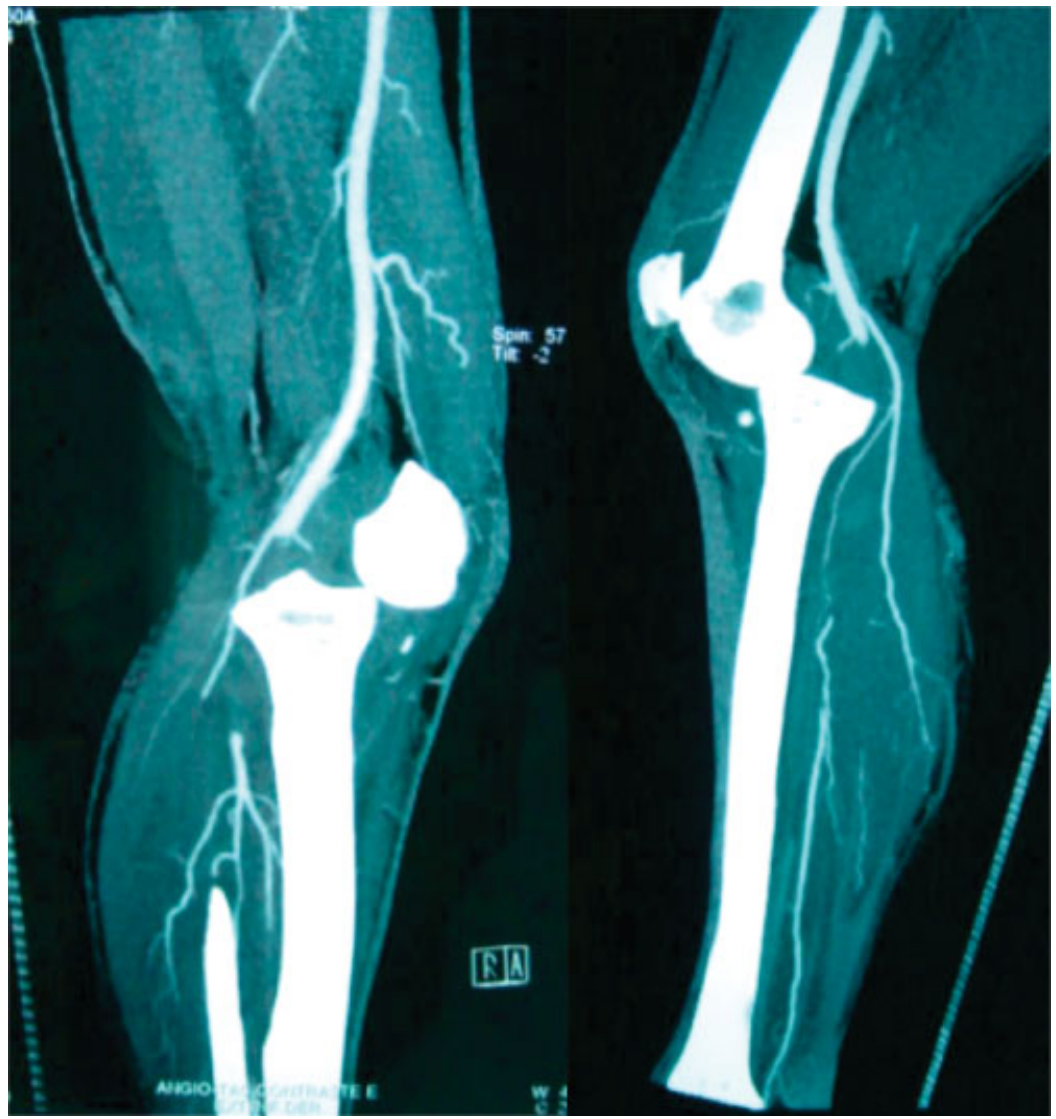

Fig. 3 AngioCT de extremidades inferiores que evidencia incongruencia articular fémoro tibial e interrupción del flujo arterial a nivel poplíteo.

El compromiso del nervio peroneo común (NPC) es un factor de mal pronóstico en contexto de luxación de rodilla. ${ }^{7,12}$ Diversas publicaciones describen compromiso del NPC entre el $5 \%$ y el $20 \%$ de las luxaciones cerradas de rodilla; Owens y col., en el año 2007, publicó una serie de 28 luxaciones de rodilla y lesión del NPC en el $75 \%$ de esos casos. ${ }^{10}$ Los escasos reportes de series que agrupan solo luxaciones expuestas, describen presencia de esa lesión hasta en un 37\% de los casos. ${ }^{10,12,20,21}$ Esa lesión presenta grados significativos de recuperación en series de luxaciones cerradas de rodilla. $^{7}$ No encontramos información relativa a ese punto en luxaciones expuestas. ${ }^{10}$ La serie quirúrgica presentada duplica las cifras reportadas en otros estudios llegando al 63,6\% de los casos con lesión neurológica; esa alta incidencia puede al igual que en el caso de lesiones vasculares, atribuirse a la alta energía involucrada que determina desplazamientos óseo significativos y en forma secundaria tracción de estructuras neurovasculares. ${ }^{1,12,20,22}$

De los 7 pacientes con lesión del NPC solamente uno presentó secuelas permanentes con dolor neuropático de difícil manejo, anestesia y alteración funcional. El tratamiento definitivo fue la amputación a nivel supracondíleo. Posteriormente este paciente reinició actividad laboral de exigencia física inferior a la pre lesional.

La infección corresponde a una complicación frecuente y habitualmente grave de esas lesiones relacionada con la energía involucrada, el déficit de cobertura de partes blandas y la isquemia regional secundaria a lesiones vasculares, que contribuye a empeorar los resultados. Wright y col., reportan $42 \%$ de infecciones en una serie de luxaciones expuestas a pesar del uso de profilaxis antibiótica y repetidos aseos quirúrgicos. ${ }^{10,20}$

La totalidad de los pacientes de esta serie estuvo sujeta a compensación laboral; ese factor influye negativamente en los resultados funcionales y satisfacción de los pacientes respecto al tratamiento. ${ }^{23}$ Esa condición sumada a la gravedad de las lesiones descritas puede explicar los bajos resultados obtenidos en las evaluaciones funcionales aplicadas. No tenemos conocimiento de publicaciones que evalúen conjuntamente esos aspectos, pero nos parece relevante mencionar que más de la mitad de los pacientes $(72,7 \%)$ incluyendo uno sometido a amputación, se reintegraron al mercado laboral tras haber sufrido una lesión de esas características.

La aplicación de un esquema estandarizado desde la evaluación inicial puede incidir en la disminución de errores durante las etapas del proceso de atención y consecuentemente en las complicaciones atribuibles a éstos; por esa razón, ese esquema debe ser sometido a revisión permanente y actualización en caso necesario. A pesar de ello, los resultados no son óptimos lo que puede explicarse por la gravedad intrínseca de ese tipo de lesiones.

Las principales limitaciones de este estudio que limitan la extrapolación de sus resultados son el diseño retrospectivo con los inherentes sesgos de memoria; la 
disparidad en los registros de la ficha clínica y ausencia de estandarización de los controles; la aplicación de escalas de evaluación funcional por vía telefónica y la baja incidencia que explica el reducido número de casos y ausencia de grupo control, elementos que limitan la extrapolación de sus resultados.

Son necesarios estudios prospectivos, multicéntricos para poder avanzar en el entendimiento de esas lesiones. Del análisis de estos datos y los disponibles en la literatura es posible concluir que la prevención es la medida con la mejor relación de costo efectividad.

\section{Conclusión}

Las luxaciones expuestas de rodilla son lesiones infrecuentes, muy complejas resultado de traumatismos de alta energía. Se asocian a severas complicaciones que pueden poner en riesgo la preservación de la extremidad y determinan pobres resultados funcionales. La estandarización mediante protocolos para su evaluación y manejo desde la evaluación inicial permite sistematizar las distintas etapas de atención, racionalizar los recursos disponibles evitando la improvisación en momentos críticos lo que podría incidir en la obtención de resultados

\section{Responsabilidades Éticas de Publicación}

Protección de las Personas y Animales

Para esta investigación no se han realizado experimentos en seres humanos ni en animales. En este artículo se obtuvo el consentimiento informado de investigación aprobado por el Comité de Ética de los pacientes que permitieron la presentación de sus fotografías y estudio imagenológico, preservando la confidencialidad de los datos.

\section{Declaración y Verificación de la Presentación}

Este trabajo no ha sido previamente publicado ni presentado simultáneamente en otra revista y tiene la autorización de los autores y su establecimiento asistencial.

\section{Bibliografía}

1 Natsuhara KM, Yeranosian MG, Cohen JR, Wang JC, McAllister DR, Petrigliano FA. What is the frequency of vascular injury after knee dislocation? Clin Orthop Relat Res 2014;472(09): 2615-2620

2 Amar MF, Chbani B, Ammoumri O, Marzouki A, Boutayeb F. Open posterior dislocation of the knee with rupture of the patellar tendon and a tibial plateau fracture. Orthop Rev (Pavia) 2010;2 (01):e7

3 Rihn JA, Groff YJ, Harner CD, Cha PS. The acutely dislocated knee: evaluation and management. J Am Acad Orthop Surg 2004;12 (05):334-346
4 Ibrahim SA, Ahmad FH, Salah M, Al Misfer AR, Ghaffer SA, Khirat S. Surgical management of traumatic knee dislocation. Arthroscopy 2008;24(02):178-187

5 Henrichs A. A review of knee dislocations. J Athl Train 2004;39 (04):365-369

6 Tod S. Physical Diagnosis of The Multiple-Ligament-Injured Knee. Clin Sport Med Vol 2000

7 Johnson ME, Foster L, DeLee JC. Neurologic and vascular injuries associated with knee ligament injuries. Am J Sports Med 2008;36 (12):2448-2462

8 Levy BA, Krych AJ, Shah JP, Morgan JA, Stuart MJ. Staged protocol for initial management of the dislocated knee. Knee Surg Sports Traumatol Arthrosc 2010;18(12):1630-1637

9 Nicandri GT, Dunbar RP, Wahl CJ. Are evidence-based protocols which identify vascular injury associated with knee dislocation underutilized? Knee Surg Sports Traumatol Arthrosc 2010;18 (08):1005-1012

10 King JJ III, Cerynik DL, Blair JA, Harding SP, Tom JA. Surgical outcomes after traumatic open knee dislocation. Knee Surg Sports Traumatol Arthrosc 2009;17(09):1027-1032

11 Chhabra A, Cha PS, Rihn JA, et al. Surgical management of knee dislocations. Surgical technique. J Bone Joint Surg Am 2005;87(Pt 1, Suppl 1):1-21

12 Peskun CJ, Chahal J, Steinfeld ZY, Whelan DB. Risk factors for peroneal nerve injury and recovery in knee dislocation. Clin Orthop Relat Res 2012;470(03):774-778

13 Fanelli GC, Orcutt DR, Edson CJ. The multiple-ligament injured knee: evaluation, treatment, and results. Arthroscopy 2005;21 (04):471-486

14 Darabos N, Gusic N, Vlahovic T, Darabos A, Popovic I, Vlahovic I. Staged management of knee dislocation in polytrauma injured patients. Injury 2013;44(Suppl 3):S40-S45

15 Seroyer ST, Musahl V, Harner CD. Management of the acute knee dislocation: the Pittsburgh experience. Injury 2008;39(07): 710-718

16 Gustilo RB, Mendoza RM, Williams DN. Problems in the management of type III (severe) open fractures: a new classification of type III open fractures. J Trauma 1984;24(08):742-746

17 Johnson ME, Foster L, DeLee JC. Neurologic and vascular injuries associated with knee ligament injuries. Am J Sports Med 2008;36 (12):2448-2462

18 Levy BA, Fanelli GC, Whelan DB, et al; Knee Dislocation Study Group. Controversies in the treatment of knee dislocations and multiligament reconstruction. J Am Acad Orthop Surg 2009;17 (04):197-206

19 Owens BD, Neault M, Benson E, Busconi BD. Primary repair of knee dislocations: results in 25 patients ( 28 knees) at a mean follow-up of four years. J Orthop Trauma 2007;21(02):92-96

20 Wright DG, Covey DC, Born CT, Sadasivan KK. Open dislocation of the knee. J Orthop Trauma 1995;9(02):135-140

21 Kadiyala RK, Ramirez A, Taylor AE, Saltzman CL, Cassell MD. The blood supply of the common peroneal nerve in the popliteal fossa. J Bone Joint Surg Br 2005;87(03):337-342

22 Prasad AR, Steck JK, Dellon AL. Zone of traction injury of the common peroneal nerve. Ann Plast Surg 2007;59(03): 302-306

23 Harris I, Mulford J, Solomon M, van Gelder JM, Young J. Association between compensation status and outcome after surgery: a metaanalysis. JAMA 2005;293(13):1644-1652 